\title{
DEVELOPMENT OF AUTOMATIC LOADING SYSTEM FOR CRUSHED STONE INDUSTRY
}

\author{
Yoshiyuki Nagano, Toru Kurenuma, Hideto Ishibashi, \\ Akira Hashimoto
Technical Research Center, Hitachi Construction Machinery Co., Ltd., 650, Kandatsu, Tsuchiura, Ibaraki, 300-0013, JAPAN

\begin{abstract}
In reply to the desire to automate one process in the work of crushing stones, an Automatic Loading System on the basis of a hydraulic excavator was developed.

The system is constituted so as to execute automatic operation relying on a Teaching Playback function. The teaching positions are only the start position for digging and the start position for loading. All desired trajectories for the required series of motion are generated on a controller.

The system was tested in the field in July 1999. The results indicated that the system was effective in stone crushing.
\end{abstract}

Keywords: Automatic Loading, Hydraulic Excavator, Teaching, Playback, Trajectory Control, Crushed Stone

\section{INTRODUCTION}

In recent years, there have been various attempts to promote automation of construction machines in mining and construction. The reasons for this are to improve productivity, safety and decrease cost of production [1].

There is also increased demand for automating work done by construction machines in the stone crushing industries. These reasons here are to decrease labor power and free skilled operators from monotonous works [2].

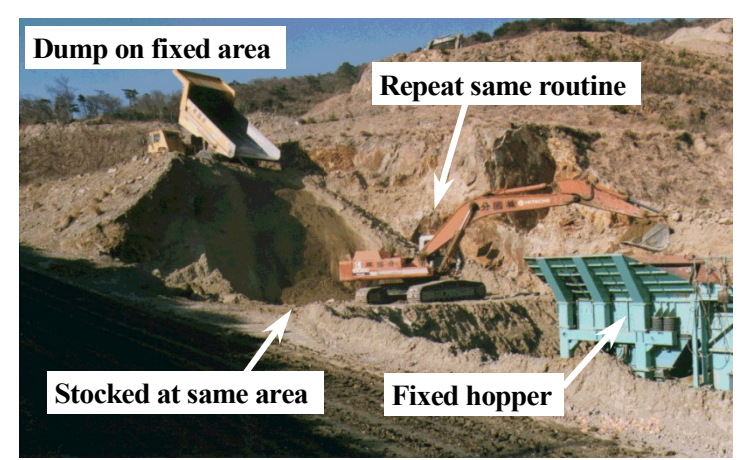

Figure 1. Crushed Stone Works

The work of hydraulic excavators in stone crushing plants is shown in Figure 1. Dump trucks dump material in one area and the material is loaded into a hopper on the crusher. The motion of the excavator is monotonous repetition. It is painfully boring work for the operator and calls out for automation. Managers of such plants can reduce worker overhead by automating this monotonous work.

In reply to this requirement, a development of an Automatic Loading System was started. It was completed recently.

In this report, the structure of the system, functions and results of testing in the field are presented.

\section{GOAL OF DEVELOPMENT}

From the client, the following conditions were provided with certain requirements.

(a) The base machine would be a hydraulic excavator of Backhoe type.

(b) The amount of production would be at least 300 ton $/ \mathrm{h}$.

Hitachi EX800H-5 Backhoe was selected for the base machine. Specifications of the excavator are shown in Table 1.

The following points were made as goals for the development.

(a) In automatic control, it would use the Teaching Playback Method.

(b) The positioning accuracy would be less than $20 \mathrm{~cm}$ to prevent collision between the bucket and hopper.

(c) The cycle time of one sequence would be equivalent to manual operating.

A system that satisfied these conditions and goals was developed. 
Table 1. Specifications of Excavator

\begin{tabular}{|c||c|c|}
\hline \multicolumn{1}{|c||}{ Model } & \multicolumn{2}{c|}{ Hitachi EX800H-5 Backhoe } \\
\hline Bucket Capacity & \multicolumn{2}{|c|}{$2.6 \mathrm{~m}^{3}(\mathrm{CECE})$} \\
\hline Operating Weight & \multicolumn{2}{|c|}{$73.2 \mathrm{t}$} \\
\hline Engine Rated Output & $440 \mathrm{PS} / 1,800 \mathrm{rpm}$ \\
\hline Overall Dimensions & Length & $14,160 \mathrm{~mm}$ \\
\cline { 2 - 3 } & Width & $4,310 \mathrm{~mm}$ \\
\cline { 2 - 3 } & Height & $4,570 \mathrm{~mm}$ \\
\hline \multirow{2}{*}{ Working Range } & Digging Reach & $13,990 \mathrm{~mm}$ \\
\cline { 2 - 3 } & Digging Depth & $8,900 \mathrm{~mm}$ \\
\cline { 2 - 3 } & Cutting Height & $12,500 \mathrm{~mm}$ \\
\hline
\end{tabular}

\section{STRUCTURE OF SYSTEM}

The structure of the system is as shown in Figure 2. The product line consists of dump trucks for carrying material, an Automatic Loading System, a crusher and a wheel loader for loading crushed stones onto the dump truck. The Automatic Loading System consists of an Automatic Loading Excavator and Playback Stand (PBS) set up far from the excavator. Control on the PBS starts and stops automatic operation, starts and stops the engine of the
Besides automatic drive, manual operation can be used. In the space behind the operating room of the excavator, the Teaching Playback Controller (TPC) and the Slip and Tilt Detecting Unit are placed. The TPC controls automatic operation. In the TPC, 3 units execute individual management. These 3 units communicate via serial communication. The Slip and Tilt Detecting Unit detects the slip of crawlers of the excavator and the tilt of the body. If either slip or tilt exceeds limit, operation is stopped. In the operating room, there are an Operational Panel and an Operational Box. In this Operational Box, a power switch, an emergency stop button and an interlock key are set. The interlock key locks the start of automatic operation. Without this key, no one can start the equipment. The Operational Panel is equipped for operation with an LCD. This shows the status of the excavator as data from angle sensors, warning messages, etc. On teaching, the data from the system can be set by operating the key according to the messages on the LCD. The operator is helped in teaching by these messages. Moreover, a Handy Operational Unit and Alarm Beeper are included. These are described in the chapter on safeguarding.

\section{FUNCTION OF SYSTEM}

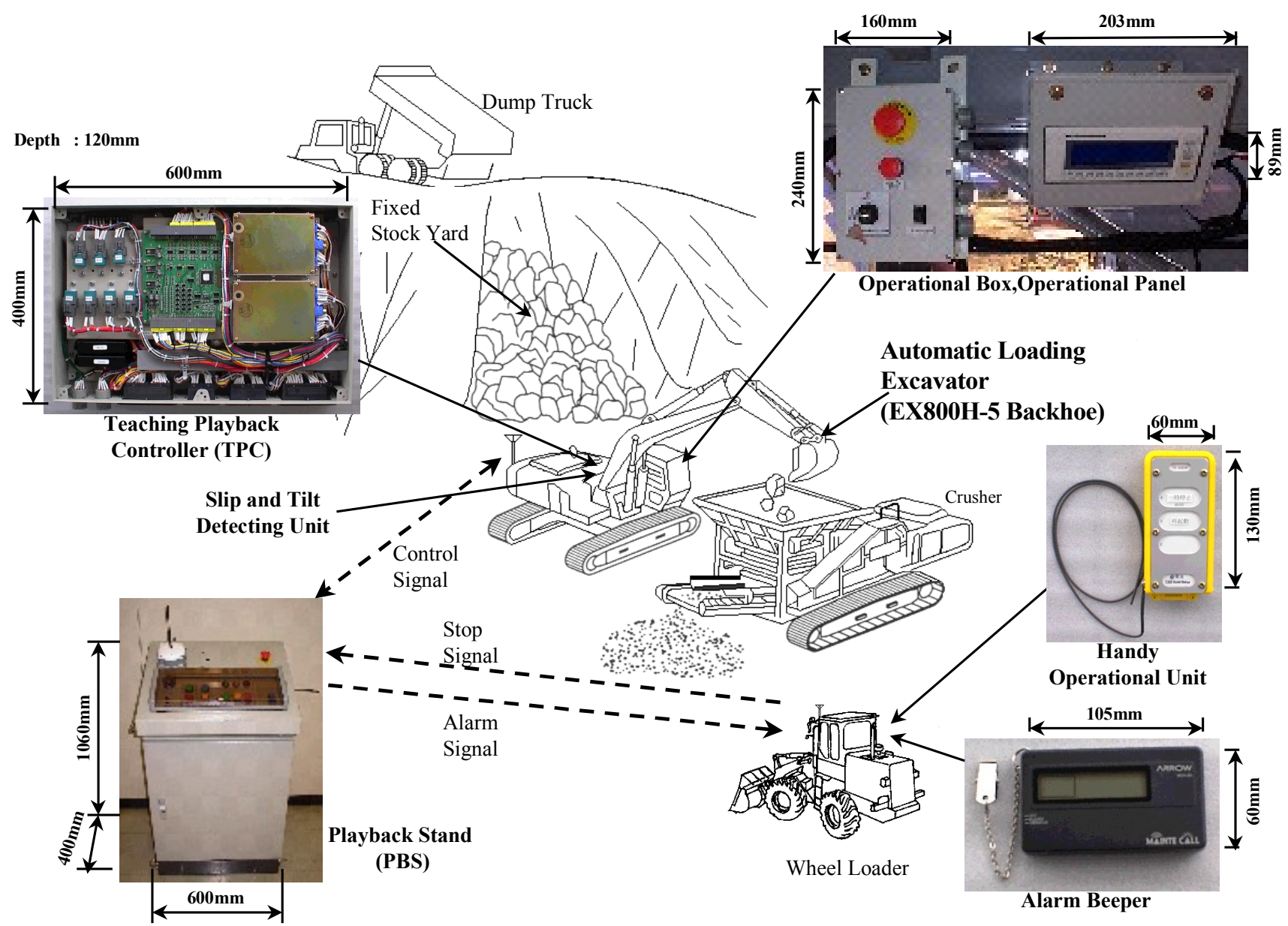

excavator and sets the engine speed.

Communication between the Figure 2. Structure of system

4.1 Teaching Playback 
In this system, the motion control method is executed by Teaching Playback. To realize this function, the TPC is divided into 3 components. These are the Teaching Playback unit, Trajectory Generator and Servo Controller. The structure is shown in Figure 3.

Teaching is started by operations on the Operational Panel. After moving the excavator to the appointed position by manual operation, the operator executes the teaching operation on the Operational Panel. On receiving the signal, the Teaching Playback unit receives data from the angle sensors attached to each axis as the set points via the Trajectory Generator and Servo Controller.

Playback is started by operation on the PBS via radio. On receiving the start signal, the Teaching Playback unit sends a set point to the Trajectory Generator. This calculates the interpolated points from receiving the set point and existing point. It then sends the interpolated points to the Servo Controller in a fixed time. The Servo Controller executes servo control by interpolation of the existing point and output control signal to the Proportional Valves. The Proportional Valves control the amount of oil flow to the actuators via the control valves. By moving the actuators, automatic operation is executed.

Figure 3. Structure of Teaching Playback

The operational procedure of the system using this Teaching Playback is shown in Figure 4. Operations are divided into executing in the operation room and executing on the PBS. In the figure, the part in the solid line is operation in the operation room and the

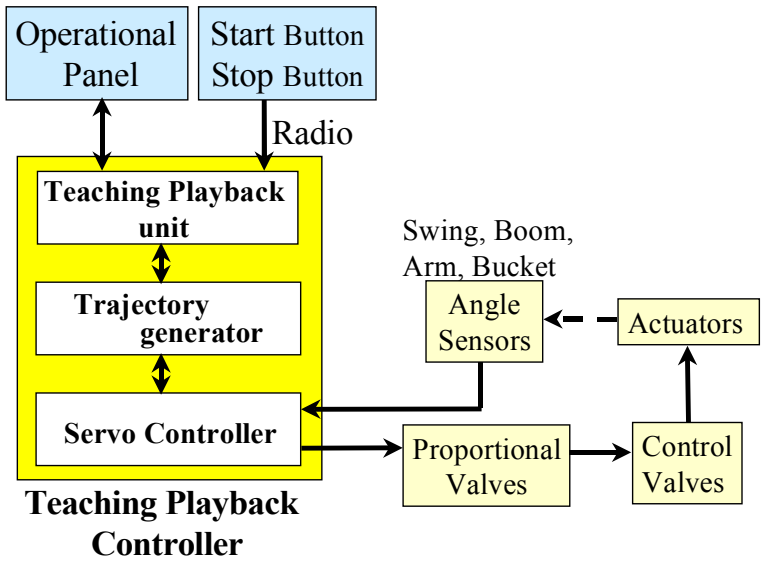

dotted line is operation on the PBS.
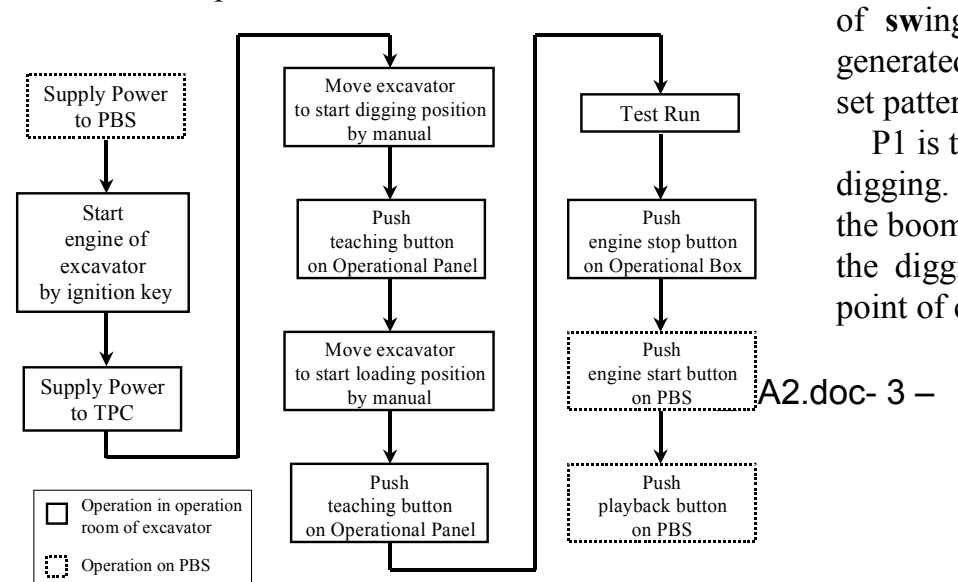

At first, the PBS supplies power. Then the operator gets on the excavator and starts the engine with the ignition key and supplies power to the TPC. Next the teaching operation is executed. In this system, the teaching points are the start point of digging and start point of loading. The operator pushes the button on the Operational Panel after moving the excavator to the start point of digging. He then pushes the button after moving to the start point of loading. This constitutes the teaching operation. After teaching, it is necessary that a Test Run be executed. The Test Run is described in the chapter on safeguarding. After the Test Run, the operator stops the engine for a moment by pushing the button in the Operational Box. The operator then gets down from the excavator. Next, he pushes the button on the PBS to start the engine and set the engine speed. Finally he pushes the button to start automatic operation.

Figure 4. Procedure of operation

\subsection{Automatic Generation of Set Points}

The teaching points and generated points are shown in Figure 5. P2 is the start point of the digging point and P7 is the start point of loading.

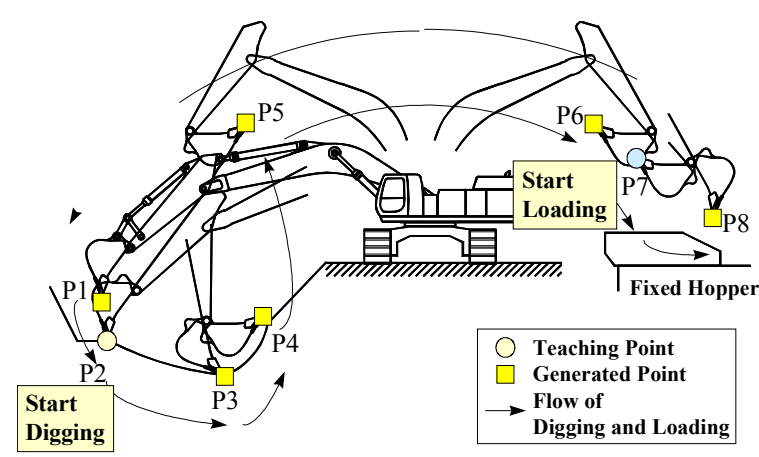

Figure 5. Teaching Point and Generated Point

These points are taught in the teaching operation. The other points are generated automatically allowing for flow of digging and loading. First the excavator digs a path from $\mathrm{p} 1$ to $\mathrm{P} 4$ and pauses at P5. Then, it swings to P6 while holding the front of excavator. Next, it loads via the path from P6 to P8 and then goes back to P1.

The method of generating the set points is shown in Table 2 . In the figure, " $\theta 2 \mathrm{sw}$ " indicates the value of swing angle sensor at P2. The set points are generated from the teaching points on the basis of the t pattern of digging and loading.

P1 is the set point for approaching the start point of digging. This point is generated as the point of raising the boom from P2. P3 is the set point in the middle of the digging motion. This point is generated as the point of crowding the arm from P2. P4 is the set point 
at the end of digging. This point is generated as the point of crowding the bucket from P3. P5 is set as a start point of swinging after digging. The purpose of generating this point is to swing safely. This point is generated from the boom, arm, bucket angles of P6 and the swing angle of P2. P6 is the set point for defending against collision between the bucket and a hopper of the crusher on swinging. This point is generated as the point of raising the boom from P7. P8 is the set point at the end of the loading. This point is generated as the point of raising the boom, dumping the arm and the bucket from P7.

Table 2. Generation of set points

\begin{tabular}{|c|c|c|c|c|}
\hline $\begin{array}{c}\text { No } \\
.\end{array}$ & Swing & Boom & Arm & Bucket \\
\hline \hline P1 & $\theta 2 \mathrm{sw}$ & $\theta 2 \mathrm{bm}+\alpha 1$ & $\theta 2 \mathrm{am}$ & $\theta 2 \mathrm{bk}$ \\
\hline P2 & $\theta 2 \mathrm{sw}$ & $\theta 2 \mathrm{bm}$ & $\theta 2 \mathrm{am}$ & $\theta 2 \mathrm{bk}$ \\
\hline P3 & $\theta 2 \mathrm{sw}$ & $\theta 2 \mathrm{bm}$ & $\theta 2 \mathrm{am}-\alpha 2$ & $\theta 2 \mathrm{bk}$ \\
\hline P4 & $\theta 2 \mathrm{sw}$ & $\theta 2 \mathrm{bm}$ & $\theta 2 \mathrm{am}-\alpha 2$ & $\theta 2 \mathrm{bk}-\alpha 3$ \\
\hline P5 & $\theta 2 \mathrm{sw}$ & $\theta 7 \mathrm{bm}+\alpha 4$ & $\theta 7 \mathrm{am}-\alpha 5$ & $\theta 7 \mathrm{bk}-\alpha 6$ \\
\hline P6 & $\theta 7 \mathrm{sw}$ & $\theta 7 \mathrm{bm}+\alpha 4$ & $\theta 7 \mathrm{am}-\alpha 5$ & $\theta 7 \mathrm{bk}-\alpha 6$ \\
\hline P7 & $\theta 7 \mathrm{sw}$ & $\theta 7 \mathrm{bm}$ & $\theta 7 \mathrm{am}$ & $\theta 7 \mathrm{bk}$ \\
\hline P8 & $\theta 7 \mathrm{sw}$ & $\theta 7 \mathrm{bm}+\alpha 7$ & $\theta 7 \mathrm{am}+\alpha 8$ & $\theta 7 \mathrm{bk}+\alpha 9$ \\
\hline
\end{tabular}

\subsection{Servo Control}

The system uses a position control and force control for the servo control. The position control operates the excavator using data from the angle sensors attached to each axis. The force control operates the excavator using data from the pressure sensors attached to each actuator. These data is combined for use so each controlled variable is regulated by gain data. Using this system, the position leads the front of the excavator along the desired paths and controls to raise the front to decrease any large load that would catch the front.

\subsection{Safeguarding}

In automatic machines, no operators are present in the vicinity of the machine to stop it in the case of an emergency. For this reason, this system needs various safeguards. Next, details of these safeguards are described as follows:

(a) The Slip and Tilt Detecting Unit is installed in the space behind the operation room of the excavator. The unit detects any slip of crawlers or tilt. If the unit detects slip exceeding a certain limit, automatic operation is stopped.

(b) After teaching, a Test Run is performed as necessary. Test Run is a function where automatic operating is executed slowly through pushing the button in the Operational Panel. Freeing the button causes automatic operating to stop. This confirms that the taught data is correct. (c) In the Operational Box, there is an engine stop button. Pushing this button stops the engine without killing the TPC. The engine start button and stop button are set on the PBS. Pushing these buttons starts and stops the engine. These functions ensure the operator can get on the excavator safely with stopping the engine.

(d) In the PBS and Operational Box, interlocking keys are placed. Setting an interlocking key at OFF prohibits start. This function protects against starting by mistake.

(e) A Handy Operational Unit to stop automatic operation and an Alarm Beeper to warn by sound and vibration is supplied. The operator carries these units so that he can manage the system from a distance.

\section{FIELD TEST}

A field test for the Automatic Loading System in a production line was executed. The conditions for this field test are shown in Figure 6.

Figure 6. Field Test

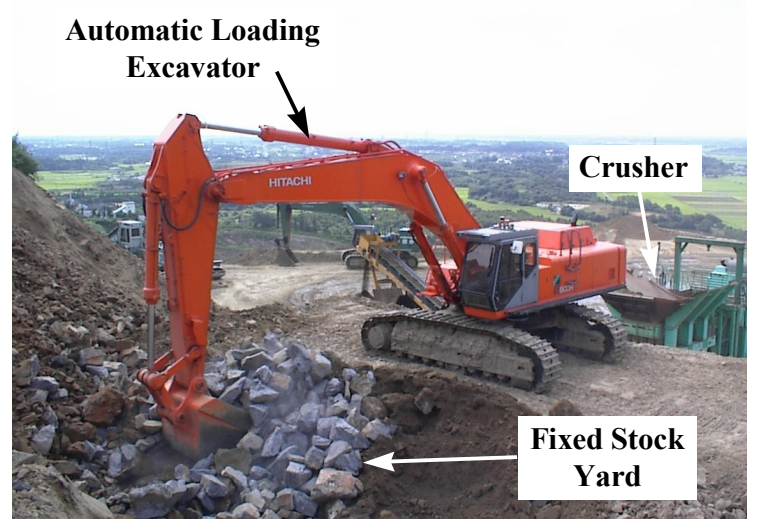

In the production line, a stockyard and a crusher were set at an angle of 180 degrees. In the center, the excavator was set 3.4 meters higher than the crusher to aid loading. By executing a continuous run with the system, we confirmed its effectiveness in the production line.

The cycle time and loaded weight in the test are shown in Table 3 . The cycle time was decided on the time for one sequence of motion of the excavator, i.e. digging, turning to the hopper, loading the hopper and turning back to the digging point. These data include the time taken for one motion which is actually slowed down to accommodate the crush speed.

The decision to reduce the amount of stone loaded in the bucket in one scoop was a preventive measure to avoid the possibility of stones dropping out and damaging the hydraulic pipes around the crusher.

Table 3. Results of Field Test 


\begin{tabular}{|c|c|}
\hline Item & Result \\
\hline \hline Cycle Time & $80 \mathrm{sec}$ \\
& (includes $\quad$ setting up time) \\
\hline Loaded Weight & 246 ton/h \\
\hline
\end{tabular}

\section{REFERENCES}

[1] S. Singh. "State of the art in Automation of Earthmoving" Journal of Aerospace Engineering, pp. 179-188. 1997.

[2] K. Miyake et al. "Development of Automatic Technology of Drilling, Loading and Hauling Operation for Crushed Stone Industry" Shigen-toSozai, Vol.112 p571-580. 1996.

\section{CONCLUSION}

(a) An Automatic Loading System for stone crushing industry was developed.

(b) Motion control was achieved using Teaching Playback.

(c) The Teaching method generated all the set points from start point of digging and start point of loading.

(d) In a field test, a good result with regard to the effectiveness of the system in a production line was verified. 